\title{
Microstructure and Mechanical Properties of In Situ Streptococcus mutans Biofilms
}

\author{
Michael S. Waters, ${ }^{\dagger, \ddagger}$ Santanu Kundu, ${ }^{*},{ }^{\dagger}$ Nancy J. Lin, ${ }^{\dagger}$ and Sheng Lin-Gibson ${ }^{*}{ }^{\dagger}$ \\ ${ }^{\dagger}$ Material Measurement Laboratory, National Institute of Standards and Technology, Gaithersburg, Maryland 20899, United States \\ ${ }^{\S}$ Dave C. Swalm School of Chemical Engineering, Mississippi State University, Mississippi State, Mississippi 39762, United States
}

Supporting Information

ABSTRACT: Insight into live microbial biofilm microstructure and mechanical properties and their interactions with the underlying substrate can lead to the development of new remedial strategies and/or materials. Here we report mechanical properties of dental pathogenic Streptococcus mutans biofilms, grown on a polystyrene-coated plate of a shear rheometer in physiologically relevant conditions, precisely controlled in a custom built bioreactor. In situ measurements demonstrated the importance of microstructure and composition of extracellular polymeric substances on the biofilm modulus. The biofilms behave like a weak gel with storage moduli higher than loss moduli. The simple but robust
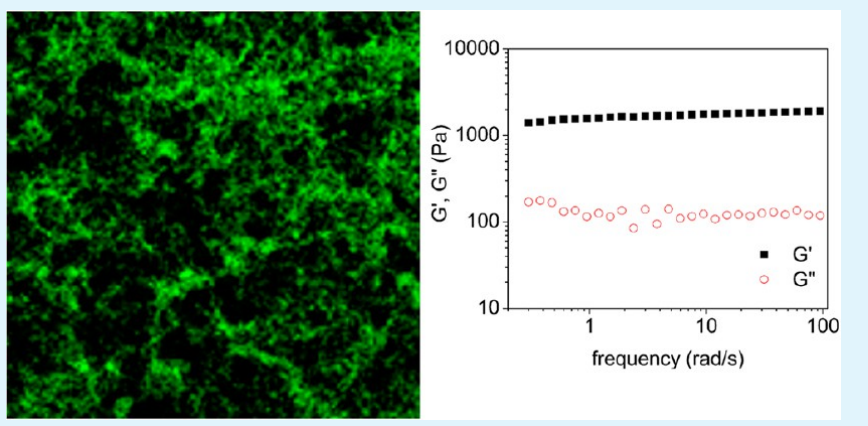
experimental technique presented here can easily be extended to other biofilm-material systems.

KEYWORDS: in situ biofilms, rheology, Streptococcus mutans, mechanical properties

\section{INTRODUCTION}

Natural persistence of microorganism-rich biofilms is necessary for many life processes; on the other hand, they can also cause significant technological or medical complications. ${ }^{1}$ Biofilms, defined here as surface-attached masses of biological material often containing a large fraction of living microorganisms, are found on nearly every surface where nutrients are available. ${ }^{2}$ The exceptional resilience of living biofilms is in part due to their ability to maintain physical structures that can withstand external chemical and mechanical challenges while permitting sufficient nutrient flow within the biofilms. ${ }^{2,3}$ Additionally, living biofilms have the capacity to alter their chemistry in response to external stimuli allowing them to modify their microstructural, mechanical, and/or adhesive properties, further facilitating survival and proliferation. ${ }^{2,3}$ Bacteria possess a wide range of sensory systems, which rapidly cause dramatic changes that can greatly influence their structural properties both as an individual bacterium and as a component to a biofilm community (e.g., temperature, electron acceptor availability, nutrient availability). Consequently, the ability to measure biofilm microstructure and mechanical properties in situ will provide fundamental insight into their responses to their environment, including the underlying substrate.

Understanding the mechanical properties of biofilms grown on specific surfaces is of great interest for various commercial applications, particularly those related to medical devices. ${ }^{4,5}$ For instance, a large percentage of infectious disease is mediated through biofilm-tissue interactions. ${ }^{4,6}$ Significant recent efforts have been devoted to determine structural and mechanical information of biofilms, with the intent of designing improved materials that could alter bacteria/biofilm response. ${ }^{5,7}$ The capacity to capture information about live, growing bacterial biofilms in the process of fouling a material of interest holds potential for the development of anti-fouling materials or for controlling biofilm-material interactions.

Bacterial biofilms are also an important and persistent component of the oral environment. ${ }^{8}$ As with all biofilms, bacterial survival within these biofilms relies heavily on their ability to sense and respond to environmental cues, which allows them to withstand various forces and maintain adhesion to the tooth surface. ${ }^{8,9}$ Streptococcus mutans (S. mutans), is one of the primary etiologic agents of tooth decay and primarily survive on surfaces within the oral cavity including the tooth surface and polymeric dental materials by adjusting their chemical and mechanical properties. ${ }^{9,10}$ S. mutans biofilms are comprised of many intertwined cells adhered together by extracellular polymeric substances (EPS; primarily a mixture of polysaccharides, DNA, degraded cellular debris) that support secondary architectures (Figure 1). Modulations of components described in Figure 1 act in concert to define the bulk mechanical properties of $S$. mutans biofilms.

The prolific nature and broad impact of biofilms have garnered much interest in measuring their microstructural and mechanical characteristics. Among various approaches, confocal microscopy has become a common method for studying threedimensional (3D) biofilm microstructure heterogeneity.

Received: October 3, 2013

Accepted: December 19, 2013

Published: December 19, 2013 


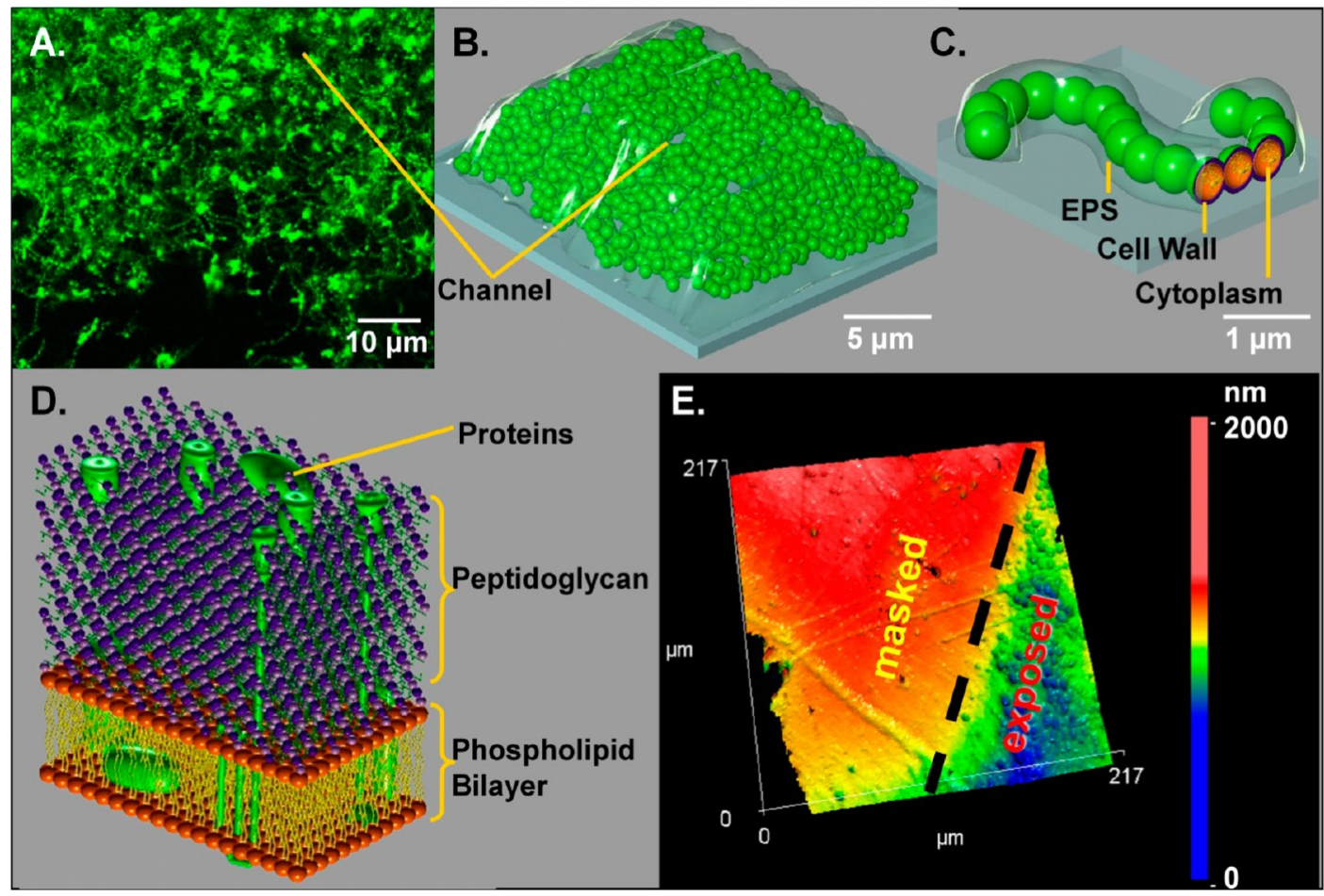

Figure 1. Description of the chemical and structural attributes influencing the mechanical properties of biofilms. (A) Confocal image of a DNAstained S. mutans biofilm grown in the presence of sucrose. The brightest spots within the image are bacterial clusters and the segmented strings are individual bacteria, which together make the biofilm. (B) Model of a S. mutans biofilm. The clear coating represents extracellular polymeric substance (EPS). (C) Each streptococcus bacterium that makes up the biofilm has a highly adhesive coating of EPS made primarily of secreted glucans. The cross-sectioned cocci depict the structural makeup of an individual cell. (D) Model depicting the cell wall composition. Peptidoglycan comprises Nacetyl glucosamine and $\mathrm{N}$-acetyl murine, linked by $\beta-1,4$ glycosidic bonds and peptide bridges. (E) 3D heat map of $S$. mutans biofilm-mediated human tooth enamel erosion, measured by interferometric optical profilometry. Tooth enamel surface was masked (left) and exposed (right) to a 24 $\mathrm{h}$ sucrose-fed S. mutans biofilm, which was removed revealing the biofilm-decayed enamel (right). The contrasting circular structures visible in the exposed area are enamel rods.

Techniques for determining the mechanical properties of biofilms include atomic force microscopy, ${ }^{11}$ microindentation, ${ }^{12}$ particle tracking methods, ${ }^{13}$ microfluidic devices, ${ }^{14}$ and shear rheometry. ${ }^{11,15-17}$ Indentation and particle tracking methods generally provide more localized properties, whereas shear rheology provides the average properties of the entire biofilm. Shear rheology has been used to determine a threshold for disruptive debulking of live $S$. mutans biofilms under constant stress. ${ }^{16}$ Pavlovsky et al. have studied the mechanical properties of Staphylococcus epidermidis (an antibiotic resistant infectious bacteria causing skin lesions in immune compromised patients and medical device contamination) biofilm using in situ rheology. ${ }^{17}$ Using oscillatory rheology, they were able to capture the biofilm growth process.

To evaluate biofilm properties that better correlate to in vivo situations, it is critical to precisely control the environment in which a biofilm is grown and measured. The primary goal of this research is to measure mechanical properties of in situ $S$. mutans biofilms grown in a custom-designed, environmentcontrolled bioreactor. Microstructural and rheological properties of intact biofilms were also compared to those from disrupted biofilms (centrifuged/spin down biofilms that are macroscopically homogeneous) to assess contributions of secondary structures and physicochemical properties to biofilm mechanical properties.

\section{MATERIALS AND METHODS}

Bacterial Strains and Culturing. S. mutans strain UA159 starter cultures $(3 \mathrm{~mL})$ were inoculated from a single colony struck on Todd Hewitt Broth (THB; Becton Dickson) agar plates grown at $37^{\circ} \mathrm{C}$ in a $5 \%$ (by volume) $\mathrm{CO}_{2}$ in air incubator as previously described. ${ }^{18}$ Biofilms were grown within the bioreactor by inoculating $10 \mathrm{~mL}$ of sterile THB or THB with $1 \%$ sucrose by mass, $1: 100$ with a $24 \mathrm{~h}$ stationary-phase starter culture, and incubating at $37^{\circ} \mathrm{C}$ in a premixed medical gas standard STM1702 equivalent to $5 \% \mathrm{CO}_{2}$ in air at atmospheric pressure.

Cultures for spin-down disrupted biofilm measurements were grown as described above in $500 \mathrm{~mL}$ THB or THB with $1 \%$ sucrose by mass in a $1 \mathrm{~L}$ Erlenmeyer flask. The entire volume along with the surface-attached biofilm (removed by scraping) was collected by 10 min centrifugation at $560 \mathrm{rad} / \mathrm{s}(3200 \mathrm{RCF})$ at room temperature. The pellet was used for rheological experiments.

Bioreactor Assembly and Rheological Measurement. Rheological measurements were performed on an ARES rheometer using 50 $\mathrm{mm}$ parallel plate geometry. The biofilm-receiving lower plate was coated with polystyrene (via spin-coated using $1 \%$ by mass polystyrene in toluene ${ }^{19,20}$ ). The entire assembly was cleaned with water and sterilized with $100 \%$ ethanol. The zero position was determined at the experimental temperature of $37^{\circ} \mathrm{C}$.

The bioreactor was assembled surrounding the lower plate (Figure 2A). After sterile assembly and inoculation, the bioreactor was jacketed with an insulated circulating water heating coil to maintain $37^{\circ} \mathrm{C}$ and was flushed with gas. Temperature was monitored by a thermocouple contacting the bottom of the lower plate. Gas was passed through 0.2 $\mu \mathrm{m}$ filtered inlet and exhaust ports to maintain internal sterility of the bioreactor. The bioreactor was disassembled after $24 \mathrm{~h}$ of growth. Superficial media was allowed to flow into an absorbent towel, 


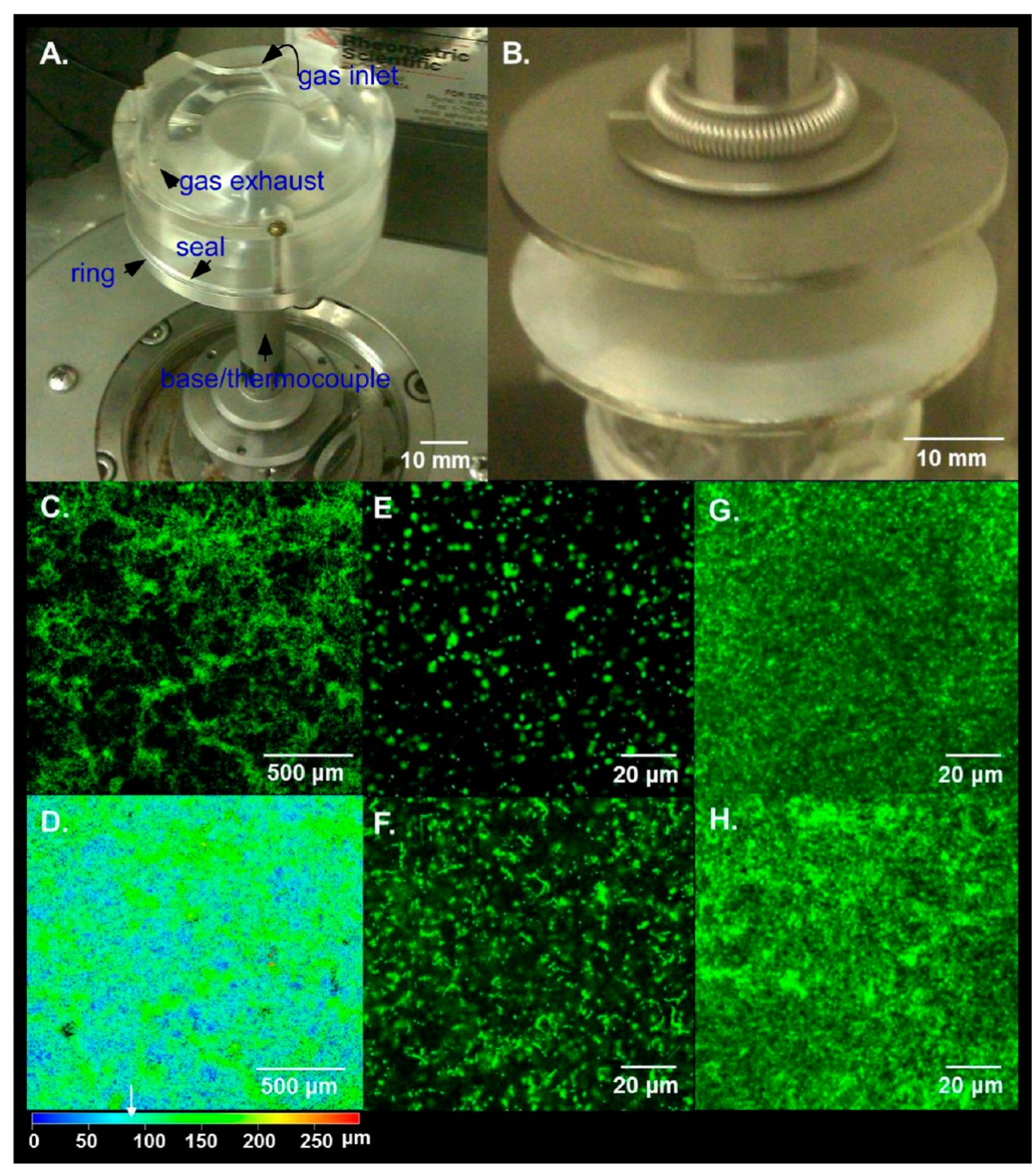

Figure 2. Bioreactor design and biofilm growth. (A) Assembled bioreactor surrounding a polystyrene-coated $50 \mathrm{~mm}$ standard disposable parallel plate, featuring gas-control and temperature feedback. (B) S. mutans biofilm (pasty cream-colored material) covered with a thin layer of media grown in the presence of sucrose on a polystyrene-coated plate directly on the rheometer, immediately before measurement. (C, D) Confocal measurement to determine gap parameters for parallel-plate rheological measurement, highlighting (C) microstructural features such as the ridges (brighter regions) and channels (darker regions) and (D) a height heat map of the confocal stack determining gap settings for biofilm measurement (arrow; $\sim 80 \mu \mathrm{m}$ ), balancing full upper plate contact with the least biofilm structural damage. (E, F) Confocal images of DNA-stained S. mutans biofilms grown directly on the rheometer in (E) the absence of sucrose and (F) presence of sucrose. (G, H) Confocal images of centrifuged (spin-down) DNA-stained S. mutans biofilms grown in $(\mathrm{G})$ the absence of sucrose and $(\mathrm{H})$ the presence of sucrose.

revealing the biofilm (Figure 2B). The upper plate was brought into contact with the biofilm by gradually lowering to $(80 \pm 1) \mu \mathrm{m}$ gap. To minimize the effect of compression as the upper plate is brought in contact with the biofilm, the upper plate was moved very slowly to dissipate the compressive stress. The compressive stress value was constantly monitored during the process. A layer of low-viscosity oil (Nikon type A immersion oil) was wiped around the edge of the gap to reduce desiccation. The rheometer oven was then rapidly closed and was brought back to $37{ }^{\circ} \mathrm{C}$. The time between disassembly and measurement was less than $2 \mathrm{~min}$.

Strain and frequency sweep experiments were performed on fresh samples. Strain sweeps were performed at strains from 0.1 to $100 \%$ and at a constant frequency of $6.28 \mathrm{rad} / \mathrm{s}$. Frequency sweeps were performed at a constant strain of $0.3 \%$, which falls in the linear viscoelastic region, in the frequency range of $0.3-100 \mathrm{rad} / \mathrm{s}$. At least three separate biofilms were evaluated in situ.

Spin-down pellets of bacteria were applied directly to the lower 50 $\mathrm{mm}$ polystyrene-coated plate with a spatula as quickly and evenly as possible. Measurements of spin-down biofilms were performed using $\sim 400 \mu \mathrm{m}$ gap with oil applied as described above to prevent desiccation. Measurements of spin-down biofilms were repeated with varying gap sizes and results were found to be independent of the gap. The experiment was repeated at least three times each time with a fresh sample.

Confocal Biofilm Characterization. Confocal images and measurements were acquired on a Zeiss LSM 510 confocal microscope and analyzed using Zeiss LSM Image Browser software. S. mutans biofilms that were grown for $24 \mathrm{~h}$ were stained for $10 \mathrm{~min}$ with fluorescent DNA stain SYBR Green (Invitrogen) and imaged as previously described. ${ }^{18} \mathrm{Z}$-stacks at $10 \mu \mathrm{m}$ and $1 \mu \mathrm{m}$ intervals covering the entire fluorescent depth were acquired using $5 \times$ and $40 \times$ objective lenses to evaluate distributions of biofilm height and structural features. These images indicated that an $80 \mu \mathrm{m}$ gap provides near $100 \%$ contact between the biofilm and the top plate.

Measurement of Biofilm-Mediated Tooth Dissolution. To confirm pathogenicity, cultures of $S$. mutans UA195 were prepared and incubated in the same conditions described above together with polished, partially masked human tooth enamel chips for $24 \mathrm{~h}$, in the 
A.

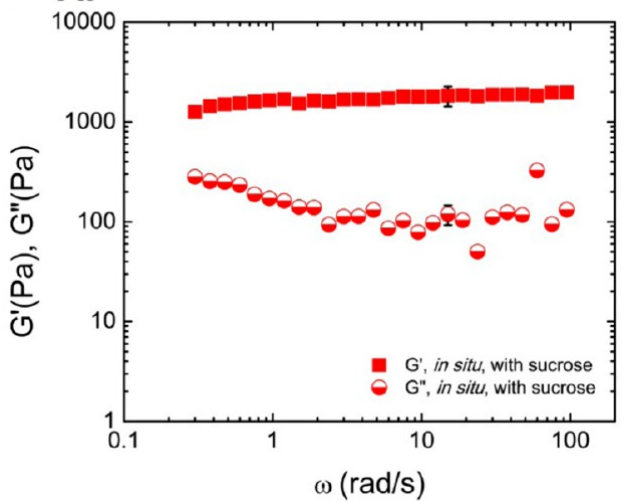

B.

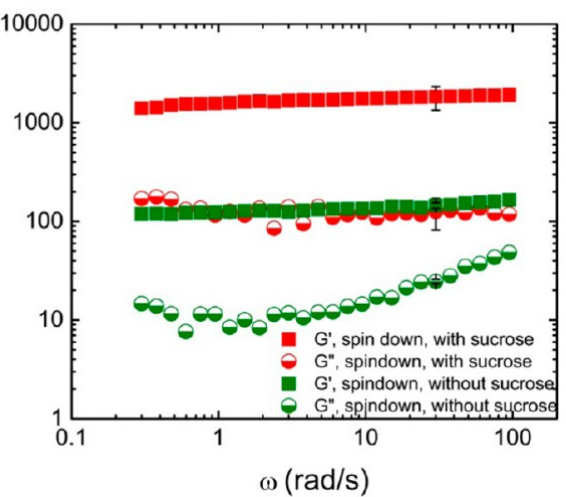

Figure 3. Storage $\left(G^{\prime}\right)$ and loss modulus $\left(G^{\prime \prime}\right)$ as a function of frequency for $(\mathrm{A})$ in situ S. mutans biofilms grown in the presence of sucrose and (B) spin-down pelleted $S$. mutans samples grown in the presence or absence of sucrose. The applied strain was $0.3 \%$.

presence of $1 \%$ sucrose by mass. Tooth preparations and interferometric optical profilometry measurements of biofilm-mediated tooth dissolution were conducted as previously described. ${ }^{21}$

\section{RESULTS AND DISCUSSION}

Bioreactor Design. The bioreactor we designed and fabricated (Figure 2A) for this study features an environmental control unit enclosing the lower plate of the rheometer. This set up permits the measurement of biofilms grown directly on the lower plate with increased measurement confidence for the following reasons. The design allows precise gap control, since the gap is calibrated within the instrument just prior to biofilm growth. Precise gap control is important for the measurements of thin biofilms. The in situ biofilm structure is highly nonuniform in the vertical direction (i.e., the biofilm becomes less dense further away from the substrate;see Figure S2 in the Supporting Information). As evident from Figures 2 and Figure S2 in the Supporting Information, over a dimension of a few square millimeters, the height of the $S$. mutans biofilm varies in some extent. To determine the height-variation, we captured images of biofilm using confocal microscopy at different depths (Z-profile). We observed plume as high as $270 \mu \mathrm{m}$ and some biofilm growth up to a height of $170 \mu \mathrm{m}$. However, most of the biofilm has a thickness of approximately $\geq 80 \mu \mathrm{m}$, which is apparent in Figure 2 and Figure $\mathrm{S} 2$ in the Supporting Information in complete coverage of image area of the biofilm. Hence, we used a gap of $80 \mu \mathrm{m}$ for all rheological studies. Measurements using a larger gap did not generate meaningful data due to insufficient contact between cellular materials and upper plate; measurements using smaller gaps compressed the biofilm, which also introduced errors. As biofilms are grown in situ, perturbation of biofilms was minimal. Gas is designed to gently circulate within the headspace, ensuring an even turnover for stable absorbance within the media without agitation. The heated jacket covering the bioreactor contacts the thermoconductive aluminum base, ensuring temperature stability for the duration of the experiment. This bioreactor design allow for facile control of conditions physiologically relevant to bacteria (e.g., nutrient medium, gas, temperature, interacting surface, etc.).

This design platform is also versatile for studying biofilm interactions with various materials. In this study, polystyrene was selected as the model surface as it is nonreactive and the most commonly used surface on which to evaluate biofilms. ${ }^{22}$ Similar techniques can be used to coat the bottom plate with a broad range of materials, including polymers, ceramics, metals, and biological materials.

Biofilm Characterization. S. mutans biofilms cultivated on the prophylactic polystyrene surface were grown either with or without sucrose supplementation in commercially available group-A streptococcal media (THB) at temperatures and gas conditions similar to the environment within the mouth, demonstrating growth consistent with previous studies. ${ }^{18} \mathrm{~S}$. mutans biofilms grown in the presence or absence of sucrose have distinctly different morphologies. Confocal measurements reveal that $S$. mutans biofilms grown in the absence of sucrose lacked clear bridge structures between the bright punctate clusters of aggregated cells, contrasted by a black background (areas lacking DNA/bacteria), giving the biofilm a more dispersed appearance (Figure 2E). Conversely, biofilms grown in presence of sucrose (Figure 2C, D, F) have a distinct and heavily interconnected structural network showing linkage between filamentous and aggregated bacterial structures while maintaining a porous network structure, reminiscent of a heterogeneous polymeric gel, permitting flow of nutrients and other important survival components.

S. mutans biofilms were also grown under similar conditions (with and without sucrose) and then centrifuged to produce spin-down cell pellets for evaluating the effect of physicochemical composition on mechanical properties. After centrifugation and pellet formation, secondary structural features within the biofilms (e.g., channels and plumes) are severely diminished, as can be seen by comparing images $\mathrm{F}$ and $\mathrm{H}$ in Figure 2 . Although disrupted, pelleted bacterial cultures still reveal visible structural differences, with sucrose-grown pellets containing aggregated bacterial structures and those grown without sucrose appearing more homogeneous (Figure 2G, H). The bright patches visible in images $\mathrm{G}$ and $\mathrm{H}$ in Figure 2 are due to a coalescence of green fluorescently stained bacteria. The dark patches between the bacteria are the void space that indirectly represents the level of biofilm hydration observed in the centrifuged samples. Dehydration experiments revealed that spin-down biofilms are comprised of $(89.3 \pm 2.6) \%$ and $(85.5$ $\pm 1.7) \%$ water by mass for no sucrose and sucrose-grown biofilms (see the Supporting Information, Figure S1), respectively.

The differences in microstructure between biofilms grown in the presence or absence of sucrose are a result of many complex physical and chemical interactions that are under the control of several regulatory systems. These structures are primarily 


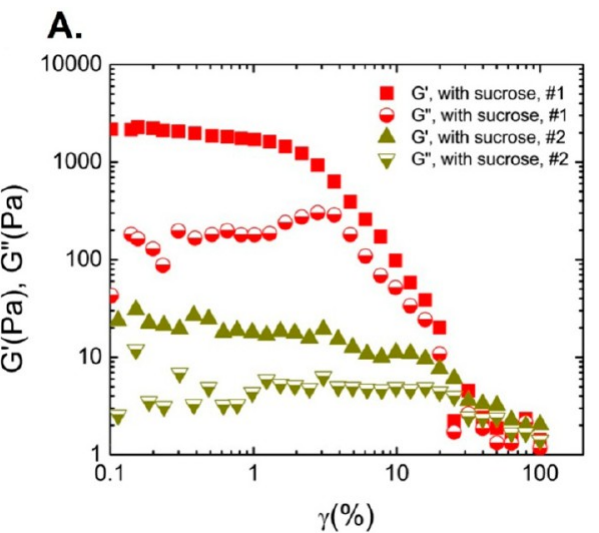

B.

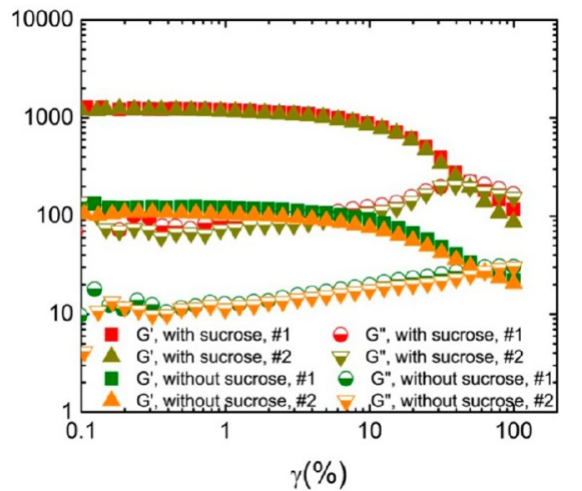

Figure 4. Reversibility of strain sweep experiments. $G^{\prime}$ and $G^{\prime \prime}$ as a function of strain for (A) in situ $S$. mutans biofilm grown in the presence of sucrose, and (B) spin-down pelleted S. mutans samples grown in the presence or absence of sucrose. The applied frequency was $6.28 \mathrm{rad} / \mathrm{s}$.

maintained and reinforced by the secretion of alpha-1,3 and alpha-1,6 glucan (polysaccharides), produced by glucosyltransferase-mediated conversion of sucrose. These polysaccharides reinforce the biofilm microstructure, converting the biofilms to rigid, desiccation-tolerant structures that can firmly attach to the tooth surface. The heat map shows that biofilm plumes reached as high as $270 \mu \mathrm{m}$ and the thickness of the confluent region of the biofilm from the polystyrene surface to the base of the plumes was $\sim 80 \mu \mathrm{m}$ (Figure $2 \mathrm{D}$ and Figure S2 in the Supporting Information). As complete contact between the sample and the top plate is important in obtaining meaningful and reproducible rheological results, $80 \mu \mathrm{m}$ was selected for all in situ sucrose-fed $S$. mutans biofilm measurements.

Upon intake, $S$. mutans also metabolizes sucrose through a series of enzymes, producing lactic acid as a by-product. The acid reduces the $\mathrm{pH}$ of the overall environment to give $S$. mutans a competitive edge over acid-intolerant bacteria, and allows the biofilm to form a protective pocket into the tooth enamel surface. 9 ,23 To confirm that the sucrose growth conditions were indeed pathogenic, in situ biofilms were probed for their pathology to human tooth enamel by interferometric optical profilometry. Within the $24 \mathrm{~h}$ culture period, the environmental $\mathrm{pH}$ was reduced from $\mathrm{pH} 7.01$ to $\mathrm{pH}$ 4.02. When a premeasured flat enamel surface was masked on one side and left exposed to biofilm growth on the other, dramatic effects were observed with respect to the dissolution of the tooth enamel (Figure 1E). These evaluations confirmed that the strain of $S$. mutans evaluated here retains its pathogenicity in its sucrose fed state.

Rheological Characterization of S. mutans Biofilms. The storage modulus $\left(G^{\prime}\right.$, representing the elastic component) is consistently higher than the loss modulus $\left(G^{\prime \prime}\right.$, representing the viscous component) over the frequency range tested for in situ biofilms grown in the presence of sucrose (Figure 3A). These biofilms behaved like a weak gel $\left(G^{\prime}>G^{\prime \prime}\right)$ and are relatively frequency independent over the entire frequency range probed. Such behavior is typical of soft elastic materials and signifies the presence of a network structure consistent with the confocal images of sucrose biofilms. Moduli of biofilms grown in situ without sucrose could not be measured, likely because of their low rigidities/interconnectivities.

Rheology measurements for centrifuged biofilms grown with and without sucrose also showed $G^{\prime}$ greater than $G^{\prime \prime}$ and were relatively frequency-independent over the entire frequency range (Figure 3B). Although a similar rheological response was observed for in situ biofilm and spin-down sucrose biofilm, the two samples were drastically different in their hydration state. The in situ biofilm contained significantly more water than the spin-down pellets as shown by the confocal images (Figure 2), which indicates that the fluid-filled interstitial space between in situ sucrose-fed S. mutans biofilms is much larger than sucrosefed spin-down biofilms. The enhanced mechanical property for the intact biofilm must be due to its network structure, which results in the modulus to the level comparable to a much dense material.

Comparison of spin-down sucrose and no sucrose biofilms revealed significant differences in mechanical properties, as $G^{\prime}$ values for sucrose samples are significant higher than that obtained for no sucrose samples. Confocal observations of interstitial space and desiccation mass experiments indicate that these spin-down biofilms had comparable amount of water content yet were one order of magnitude different in modulus when measured by rheology. These differences are attributed to chemical differences-the presence of large amount of rigid hydrophobic extracellular alpha-1,3 glucan, produced by sucrose-fed biofilms. ${ }^{24,25}$

Strain sweep experiments were performed to determine the linear viscoelastic region and to provide information about the large strain responses of biofilms on both in situ and spin-down biofilms. The linear viscoelastic region for in situ biofilm was up to $\sim 1 \%$ strain; further increases in strain resulted in strong strain-softening (Figure 4A). Additionally, the modulus of the in situ biofilm does not recover appreciably when the measurement was repeated on the same sample. In fact, the intact biofilm (first run) has a $G^{\prime}$ that was about two orders of magnitude higher than the disrupted biofilm (second run). This behavior is consistent with disruption of the interconnected structure at higher strains. Note that the second run was started immediately, without any wait time to avoid potential repairing of the live biofilm network.

The linear viscoelastic region extended to $\sim 10 \%$ strain for all spin-down biofilms (Figure 4B). Consistent with frequency sweep experiments, $G^{\prime}$ for biofilms grown with sucrose was higher than those grown without sucrose. The strain sweep experiments are highly repeatable when the sample is run multiple times, indicating a lack of structural change upon imposed strain. The recovery is likely due to direct measurement of the mechanical properties of the overall biomass, without significant contribution from larger structures. Interestingly, there is a $G^{\prime}$ to $G^{\prime \prime}$ crossover at higher strains 
for both spin-down samples, indicating the material will become more viscous-like under large strain deformations. The moduli were quite different for spin-down samples grown with or without sucrose despite the fact that the interstitial spaces between the bacteria have been largely removed for both samples. The large difference in $\mathrm{G}^{\prime}$ indicates that the components holding these bacteria together are quite different in composition and/or distribution. As described above, the shifting of EPS properties by secretion of extracellular glucan via the metabolism of sucrose by glucosyltransferase enzymes is expected to be the most important reason for this modulus shift. $^{24,25}$

\section{CONCLUSIONS}

In this study, we demonstrate that oscillatory shear rheology can be used to evaluate in situ mechanical properties of live acidogenic $S$. mutans biofilms. Biofilms were grown directly within the rheometer on a standard plate coated with a material of our choosing, in a custom bioreactor that acts as a standalone, environmentally controlled culturing system. This approach eliminates mechanical damage inherent to transferring biofilms on plates to the rheometer and greatly reduces the time between culturing and measurement to provide higher degrees of repeatability. We show that the biofilm microstructures play a key role in the overall mechanical properties, i.e., disrupted biofilm due to shear reduced $G^{\prime}$ by approximately two orders of magnitude. We were also able to discern $G^{\prime}$ differences due to biofilm EPS variations. This method to measure biofilm-material interactions can be easily adapted to most biofilms or materials for advancing medical or environmental applications, particularly in the development of materials that modify biofilm interactions.

\section{ASSOCIATED CONTENT}

\section{S Supporting Information}

Water retention of biofilms, confocal micrographs, and heterogeneity of biofilms are included in the Supporting Information. This material is available free of charge via the Internet at http://pubs.acs.org/.

\section{AUTHOR INFORMATION}

\section{Corresponding Authors}

*E-mail: santanukundu@che.msstate.edu.

*E-mail: slgibson@nist.gov.

\section{Present Address}

${ }^{\ddagger}$ M.S.W. is currently at Office of In Vitro Diagnostics and Radiological Health, Center for Devices and Radiological Health, FDA, Silver Springs, MD 20993.

\section{Notes}

The authors declare no competing financial interest.

\section{ACKNOWLEDGMENTS}

This work was in part supported by a National Research Council (NRC) ARRA postdoctoral Fellowship and an Interagency Agreement between NIDCR/NIH and NIST [Y1-DE-7005-01]. This is an official contribution of the National Institute of Standards and Technology, and is not subject to copyright in the United States. Certain commercial equipment, instruments, or materials are identified in this paper in order to specify the experimental procedure adequately. Such identification is not intended to imply recommendation or endorsement by the National Institute of Standards and
Technology, nor is it intended to imply that the materials or equipment identified are necessarily the best available for the purpose.

\section{REFERENCES}

(1) Community Structure and Co-Operation in Biofilms; Allison, D. G., Gilbert, P., Lapin-Scott, H.M., Wilson, M., Eds.; Society for General Microbiology Symposia; Cambridge University Press: Cambridge, U.K., 2000.

(2) Davey, M. E.; O'toole, G. A. Microbiol. Mol. Biol. Rev. 2000, 64, $847-867$.

(3) Nadell, C. D.; Xavier, J. B.; Foster, K. R. FEMS Microbiol. Rev. 2009, 33, 206-224.

(4) Bryers, J. D. Biotechnol. Bioeng. 2008, 100, 1-18.

(5) Biofilms in Medicine, Industry and Environmental Biotechnology: Characteristics, Analysis and Control; Lens, P., O'flaherty, V., Moran, A. P., Stoodley, P., Mahony, T., Eds.; International Water Association: London, 2003.

(6) Wolcott, R; Dowd, S. Plast. Reconstr. Surg. 2011, 127 (Suppl 1), 28S-35S.

(7) Houari, A.; Picard, J.; Habarou, H.; Galas, L.; Vaudry, H.; Heim, V.; Di Martino, P. Biofouling 2008, 24, 235-240.

(8) Bowden, G. H. W.; Hamilton, I. R. Crit. Rev. Oral. Biol. Med. 1998, 9, 54-85.

(9) Nobbs, A. H.; Lamont, R. J.; Jenkinson, H. F. Microbiol. Mol. Biol. Rev. 2009, 73, 407-450.

(10) Klein, M. I.; Duarte, S.; Xiao, J.; Mitra, S.; Foster, T. H.; Koo, H. Appl. Environ. Microbiol. 2009, 75, 837-841.

(11) Lau, P. C. Y.; Dutcher, J. R.; Beveridge, T. J.; Lam, J. S. Biophys. J. 2009, 96, 2935-2948.

(12) Cense, A. W.; Peeters, E. A. G.; Gottenbos, B.; Baaijens, F. P. T.; Nuijs, A. M.; van Dongen, M. E. H. J. Microbiol. Methods 2006, 67, 463-472.

(13) Cheong, F. C.; Duarte, S.; Lee, S.-H.; Grier, D. G. Rheol. Acta 2009, 48, 109-115.

(14) Hohne, D. N.; Younger, J. G.; Solomon, M. J. Langmuir 2009, $25,7743-7751$.

(15) Lieleg, O.; Caldara, M.; Baumgärtel, R.; Ribbeck, K. Soft Matter 2011, 7, 3307-3314.

(16) Vinogradov, A. M.; Winston, M.; Rupp, C. J.; Stoodley, P. Biofilms 2004, 1, 49-56.

(17) Pavlovsky, L.; Younger, J. G.; Solomon, M. J. Soft Matter 2013, 9, 122-131.

(18) Waters, M. S. Ph.D. Dissertation, University of Southern California, Los Angeles, CA, 2009.

(19) Stafford, C. M.; Guo, S.; Harrison, C.; Chiang, M. Y. M. Rev. Sci. Instrum. 2005, 76, 062207-1-062207-5.

(20) Torres, J. M.; Stafford, C. M.; Vogt, B. D. Polymer 2010, 51, 4211-4217.

(21) Waters, M. S.; Yang, B.; Lin, N. J.; Lin-Gibson, S. In Optical Measurements, Modeling, and Metrology; Proulx, T., Ed.; Springer: New York, 2011; Vol. 5, pp 337-344.

(22) Li, Y.-H.; Lau, P. C. Y.; Tang, N.; Svensäter, G.; Ellen, R. P.; Cvitkovitch, D. G. J. Bacteriol. 2002, 184, 6333-6342.

(23) Nicolas, G. G.; Lavoie, M. C. Can. J. Microbiol. 2011, 57, 1-20.

(24) Hamada, S.; Kobayashi, Y.; Slade, H. D. Microbiol. Immunol. 1978, 22, 279-282.

(25) Mukasa, H.; Slade, H. D. Infect. Immun. 1973, 8, 555-562. 